\title{
A indústria de defesa no Brasil: investigando a competência essencial
}

\author{
The defense industry in Brazil: \\ researching the core competence
}

Rev. Bras. Est. Def. v. 5, nº 2, jul./dez. 2018, p. 87-111

DOI: $10.26792 /$ RBED.v5n2.2018.75025

ISSN 2358-3932

\section{WANDICK LEÃO \\ JULIANA BONOMI SANTOS \\ CAIO SOUSA DA SILVA}

\section{INTRODUÇÃO}

Produtos militares, como mísseis, mostram crescimento da demanda em torno de $5 \%$ ao ano, sendo que agora eles têm mais computadores do que explosivos, logicamente para encontrar o alvo de forma mais precisa e autônoma (The Economist 2016). Desenvolver e fornecer produtos como esses não é simples, pois a Base Industrial de Defesa (BID) é repleta de características singulares que podem prejudicar as empresas que atuam nesse segmento (Cunha and Amarante 2011; Markusen 1986).

Os contratos de fornecimento, geralmente concebidos junto aos governos nacionais, podem tanto prover uma base segura para o desenvolvimento de novos produtos e serviços, como sofrer demasiada pressão por parte de intervenções públicas, atrapalhando questões internas das empresas, por exemplo (p. ex.) planejamento operacional e financeiro.

Além disso, com o progresso técnico dos armamentos, a BID se especializou e ganhou dinâmica própria, ampliando cada vez mais sua demanda por equipamentos militares avançados, muitos dos quais no "estado da

\footnotetext{
Wandick Leão - Professor Auxiliar do Insper - Instituto de Ensino e Pesquisa. Graduado pelo Centro Universitário Metropolitano de São Paulo (UNIMESP), mestre pelo Centro Universitário FEI e doutorando em Administração de Empresas pela Escola de Administração de Empresas de São Paulo da Fundação Getulio Vargas (FGV-EAESP).

Juliana Bonomi Santos - PhD em Management Science pela Lancaster University Management School (2013) e professora do programa de pós-graduação em administração da Escola de Administração de Empresas de São Paulo (FGV-EAESP).

Caio Sousa da Silva - Mestre em Administração de Empresas pelo PPGA do Centro Universitário FEI, no tema de Internacionalização de Negócios Sociais.
} 
arte” (Agência Brasileira de Desenvolvimento Industrial [ABDI] 2011). Para se ter uma ideia desse panorama, de acordo com a ABDI (2011), essas empresas investem, em média, $10 \%$ de seu faturamento em atividades de inovação. Esse tipo de investimento acarreta consequentemente a demanda de uma mão de obra mais qualificada, comprovada pela presença de mais engenheiros, tecnólogos, mestres/doutores do que em Outras Indústrias (OI). Pesquisas realizadas por Caldwell e Howard (2014) e Rauen (2015), discutem em contextos distintos, os desafios inerentes à BID, evidenciando que empresas que atuam nessa indústria necessitam de competências organizacionais especificas e com elevado nível de desenvolvimento.

Dessa forma, as empresas da BID parecem necessitar ou deter competências organizacionais mais avançadas do que as das empresas de OI. Só assim seria possível sobreviver e prosperar nesse ambiente complexo, incerto e dependente de inovação. Além disso, é possível que as empresas da BID tenham competências específicas mais avançadas, dado o aprendizado imposto pelo setor em que atuam. Com o tempo, essa competência pode se tornar essencial, sendo fator de vantagem competitiva nesse mercado.

Diante desse quadro, é importante avaliar empiricamente se o nível de desenvolvimento das competências das empresas da BID se diferem de empresas de OI. Em caso afirmativo, importa entender como as dificuldades da indústria moldam as competências das empresas da BID (Ferreira 2016), identificando quais delas são mais ou menos desenvolvidas — p. ex., a gestão de projetos. Afinal, essa indústria tem como essência a produção de equipamentos tecnológicos e complexos, desenvolvidos em longos ciclos e fabricados sob encomenda (Amarante 2012).

O presente artigo explora o conceito de competência sob o prisma organizacional, isto é, atividades funcionais como marketing, finanças ou operações, mas que, quando capazes de oferecer diferenciação e vantagem competitiva diante de outras empresas e indústrias, podem ser percebidas como essenciais (Prahalad and Hamel 1990). Esse artigo realiza um estudo exploratório e quantitativo, com foco na identificação e análise das competências de empresas fornecedoras, tanto da BID como de OI (agricultura, têxtil, metalurgia, construção civil e outras). Buscando como principais achados: I) os níveis das competências das empresas fornecedoras da BID e de OI; e II) apontar em quais competências as empresas fornecedoras da BID estão em situação superior ou inferior em relação às empresas de OI.

Dessa forma, essas análises proporcionam informações que podem ser aproveitadas por ambos os perfis de empresas, bem como pelo governo, no sentido de entender em quais esferas é necessário aprimoramento. Para a coleta de dados, foi empregado um survey com duas amostras distintas de empresas (BID x OI). Com essa abordagem, a análise de dados buscou 
aferir diferenças entre os níveis de competências dessas amostras (Field 2009). Os resultados obtidos através de testes paramétricos (Anova) e testes post hoc sugerem que a maioria das competências das empresas de ambas as indústrias se equivalem, sendo que algumas competências são mais desenvolvidas em OI e outras na BID. Todavia, a gestão de projetos demonstra estar em um patamar superior nas empresas da BID, revelando médias superiores, tanto em termos numéricos quanto em significância estatística, resultado que, em parte, demonstra que essa competência parece ser fundamental nessa indústria.

Esses resultados indicam certa homogeneidade nas competências necessárias para se competir nas diferentes indústrias. Esse achado desmistifica a visão de que, devido à sua complexidade, são necessários maiores níveis de competências para se competir na BID. Assim, empresas da BID podem beneficiar-se da adoção de práticas gerenciais, amplamente difundidas na literatura de gestão. Além disso, o estudo contribui ao mostrar que as empresas da BID estão em defasagem em relação a outras indústrias de elevada intensidade tecnológica, indicando áreas de ação. Por fim, este trabalho indica qual a principal competência na BID — habilidade de gerir projetos. Logo, aponta uma área essencial, na qual as empresas dessa indústria devem cada vez mais investir e qualificar seus funcionários.

Este trabalho está organizado da seguinte forma: primeiramente, na segunda seção, é apresentada a revisão teórica sobre a Política de Defesa no Brasil, BID, Intensidade Tecnológica e Competências Organizacionais. Na terceira seção é discutido o método. Na quarta são analisados os dados e na última seção tem-se a discussão e as considerações finais.

\section{REVISÃO DA LITERATURA}

\section{Política de Defesa no Brasil}

Segurança é uma necessidade primária de todo país (Duarte 2012) e nenhum país pode deixar de se defender (Cunha and Amarante 2011; Peng 2004). O Brasil é um país com dimensões continentais e com a maior costa atlântica do mundo, e constitui fronteira com dez países, pouco povoadas e de difícil controle. Outrossim, tem aproximadamente 191 milhões de habitantes, sendo a quinta maior população do planeta (Livro Branco de Defesa Nacional [LBDN] 2012). Além disso, o país tem diversas riquezas em território continental e na zona econômica, como terras produtivas, florestas, rios, ilhas, jazidas e bastante água doce. Conta, ainda, com capacidade de produção de energias renováveis e não renováveis, proteínas animal e vegetal, sem falar da descoberta do pré-sal, que colocou o país em 
outro patamar (Franko 2014). Além do elevado conjunto de características internas, o país precisa estar atento a fenômenos exteriores como globalização, narcotráfico, tráfico de armas, pirataria marítima e crises econômico-financeiras, eventos que podem refletir na paz e segurança de um país (LBDN 2012).

Tais questões exigem Segurança e Defesa (Duarte 2012). Segurança significa que a sociedade e os indivíduos devem estar livres de riscos, pressões ou ameaças não militares, mas também econômica, ambiental e social (Corrêa and Cagnin 2016), e Defesa Nacional (DN) é caracterizada como o "conjunto de medidas e ações do Estado, com ênfase na expressão militar, para a defesa do território, da soberania e dos interesses nacionais contra ameaças preponderantemente externas, potenciais ou manifestas" (LBDN 2012, 24). O Brasil conta com documentos que formalizam essas questões, como a Política de Defesa Nacional (PDN) aprovada em 2005 e a Estratégia Nacional de Defesa (END) lançada em 2008, ambas atualizadas em 2012 e, recentemente, o Livro Branco de Defesa Nacional (Schimdt and Assis 2013). Já o instrumento militar brasileiro, no caso, as Forças Armadas (FA), é composto pela Marinha, Exército e Aeronáutica, organização similar a vários outros países, como na Inglaterra e China (Caldwell and Howard 2014; Peng 2004). O papel dessas instituições consiste em assegurar a integridade e a defesa do país, sendo que ambas são integrantes da estrutura do Ministério da Defesa (MD), o qual tem relevante papel de gestão e orientação (Corrêa and Cagnin 2016; Mendes 2010).

Percebe-se que os líderes brasileiros têm se preocupado com essas temáticas. Uma das preocupações, talvez esteja relacionada ao fato de que Segurança e Defesa precisam de recursos, humanos e materiais, como armas, dispositivos e equipamentos, que dada essa aplicação específica demandam sofisticação e alto teor tecnológico (Cunha and Amarante 2011; Markusen 1986), sendo esse o tipo de solução, cada vez mais procurada pelas forças armadas no mundo todo (Dunne 1995; Duarte 2012). Portanto, parece que, mais difícil do que se defender e manter uma mínima segurança, seja prover os recursos para tais necessidades. Isto é, o Brasil carece de uma BID própria e bem estabelecida, a qual envolve alta tecnologia e investimento, que considerando o contexto brasileiro trata-se de desafios notáveis e excludentes (Franko 2014).

\section{Base Industrial de Defesa (BID) no Brasil e suas particularidades}

A Indústria de Defesa existe desde o final do século XIX, sendo resultado da ascensão do capitalismo industrial, que revolucionou a tecnologia das armas, por meio da mecanização e da produção em massa (Dunne 1995). 
Define-se como Base Industrial de Defesa (BID), toda a estrutura responsável pela pesquisa, desenvolvimento, produção, distribuição e manutenção dos produtos e serviços de uso militar. Nela, se encontram as fábricas e prestadores de serviços, responsáveis pelo fornecimento de equipamentos, armas e serviços de combate ao homem contemporâneo (Amarante 2012; LBDN 2012; Markusen 1986). Esses produtos podem ser desde armas de diferentes portes e calibres, sistemas de monitoramento e combate, além de itens não letais, mas estratégicos, como veículos e combustíveis até produtos de consumo, como roupas especiais e alimentos (Dunne 1995). Para assegurar a oferta adequada desses suprimentos, este mercado necessita tanto da iniciativa privada como das instituições públicas (Amarante 2012).

O fortalecimento da BID começou em 1999 com a criação do MD; todavia, somente com a END publicada em 2008, estabeleceu-se por escrito a necessidade de uma capacitação do Brasil em relação a essa indústria (Mendes 2010). Cabe destacar que a BID tem grande importância para qualquer país que pretenda ocupar uma justa posição no cenário internacional (Cunha and Amarante 2011). A seguir, são elencados quatro motivos para se construir uma BID nacional: I) Estímulo à economia, à produção industrial e de serviços, podendo gerar inovações tecnológicas, que podem futuramente ter aplicação dual (Eliasson 2011; Graham and Hardaker 1998; Markusen 1986); II) Fornecimento de equipamentos para a defesa da nação, que de outra forma teriam de ser importados de outros países (ABDI 2011); III) Geração de emprego e renda, como ocorreu no Reino Unido no final dos anos 1990, quando essa indústria empregou cerca de 420.000 pessoas (Graham and Hardaker 1998); e IV) desenvolvimento e a capacitação de recursos humanos de alto nível (Amarante 2012).

Apesar do considerável crescimento nos últimos anos, a BID é um importante segmento econômico no Brasil há três décadas. Contudo, grande parte das empresas que compõem a BID são remanescentes de antigos e grandes projetos militares, iniciados nas décadas de 1970/1980, ou, ainda, é formada por empresas que acolheram os projetos iniciados naquela época (LBDN 2012). Esse papel de estímulo a vários outputs que a BID pode proporcionar ao país parece estar ligado a uma questão cada vez mais presente entre pesquisadores, técnicos e outros profissionais do setor, que é o fato de a BID ser bastante diferente das OI, em relação às bases científica, tecnológica, industrial e logística (Cunha and Amarante 2011; Freitas 2011).

Por outro lado, a BID sofre com diversos problemas, como: I) Fortes restrições políticas, que afetam questões comerciais e de operações, tornando os custos elevados (Amarante 2012); II) Desenvolvimento tecnológico, que depende de condições sociais e atividades produtivas, que são bem diferentes entre a BID e OI (Duarte 2012); III) Os produtos desenvolvidos 
e produzidos são destinados à proteção e combate, onde a confiabilidade é um dos requisitos fundamentais, pois, não se espera ou se pretende que haja uma segunda chance; IV) Os equipamentos, dispositivos, armas e sistemas são desenvolvidos em longos ciclos, com produção sob demanda e custo bancado pelo cliente; e V) Os produtos e serviços desenvolvidos exigem conhecimentos multidisciplinares, oriundos de vários segmentos, como mecânica, química, eletrônica, informática, oceanografia, etc., requerendo profissionais de alto nível técnico, com visão holística e competências em gestão de projetos (Cunha and Amarante 2011).

Em geral, além da gestão de projetos, as empresas da BID precisam ter outras competências funcionais bem desenvolvidas, como, produção, integração, logística, pós-venda, tecnológica, desenvolvimento experimental, pesquisa básica e formação de recursos humanos (Amarante 2012). Portanto, diante dessas considerações, propõe-se a primeira hipótese deste trabalho:

\section{H1: AS COMPETÊNCIAS DAS EMPRESAS DA BASE INDUSTRIAL DE DEFESA (BID) SÃO MAIS DESENVOLVIDAS DO QUE AS DAS EMPRESAS DE OUTRAS INDÚSTRIAS (OI)}

\section{Base Industrial de Defesa (BID) no Brasil e Intensidade Tecnológica}

Segundo Amarante (2012), a tecnologia tem invadido o cenário militar, influenciando as operações das forças armadas, que cada vez mais têm adotado novas maneiras de se fazer guerra, especialmente através de meios, como Robótica, Automação, Sistêmica e Cibernética, todas elas com alto teor tecnológico. Logo, a BID é considerada uma indústria específica por dois motivos: I) Desenvolvimento de produtos e serviços para defesa e segurança; e II) Emprego de alta intensidade tecnológica nos respectivos produtos e serviços desenvolvidos.

A intensidade tecnológica representa o emprego de conhecimento técnico aos serviços e/ou produtos em diversos setores (Chiarini and Silva 2017). No Brasil, ela é calculada através de uma metodologia criada pela Organização para a Cooperação e Desenvolvimento Econômico (OCDE), que estrutura os diferentes níveis de intensidade, através da quantidade de recursos que as empresas investem em pesquisa e desenvolvimento no decorrer dos anos. As empresas que investem mais em Pesquisa e Desenvolvimento (P\&D) e exercem atividades de cunho tecnológico em seus processos têm maior grau de inovação incorporado em seus resultados (Chiarini and Silva 2017). No Brasil, pouco se fala sobre a intensidade tecnológica das indústrias. Furtado e Carvalho (2005) apresentam infor- 
mações da OCDE para ilustrar como é estruturada a intensidade tecnológica de algumas indústrias no Brasil (ver Figura 1):

\begin{tabular}{cccc|}
$\begin{array}{c}\text { Alta intensidade } \\
\text { tecnológica }\end{array}$ & $\begin{array}{c}\text { Média-alta } \\
\text { intensidade } \\
\text { tecnológica }\end{array}$ & $\begin{array}{c}\text { Média-baixa } \\
\text { intensidade } \\
\text { tecnológica }\end{array}$ & $\begin{array}{c}\text { Baixa } \\
\text { intensidade } \\
\text { tecnológica }\end{array}$ \\
Farmacêutico & $\begin{array}{c}\text { Meículos } \\
\text { automotores }\end{array}$ & Borracha e plásticos & Madeira \\
Informática & Química & Petróleo & Papel e celulose \\
Eletrônica & Ferrovia & $\begin{array}{c}\text { Combustíveis } \\
\text { nucleares }\end{array}$ & $\begin{array}{c}\text { Editorial e gráfica } \\
\text { Telecomunicações }\end{array}$ \\
Transporte & Metalurgia & $\begin{array}{c}\text { Alimentos e } \\
\text { bebidas }\end{array}$ \\
Instrumentos & $\begin{array}{c}\text { Máquinas e } \\
\text { equipamentos }\end{array}$ & & Fumo \\
& & & Têxtil \\
\hline
\end{tabular}

Figura 1 - Indústrias por intensidade tecnológica no Brasil.

Fonte: Adaptado de Furtado e Carvalho (2005).

A intensidade tecnológica é tão essencial na BID que autores como Amarante (2012), afirmam categoricamente que essa indústria só poderá existir desde que se crie no Brasil uma infraestrutura de Ciência, Tecnologia e Inovação (C, T \& I), a qual deve ser trabalhada de modo integral, criando-se o conceito de iceberg científico (Figura 2). 


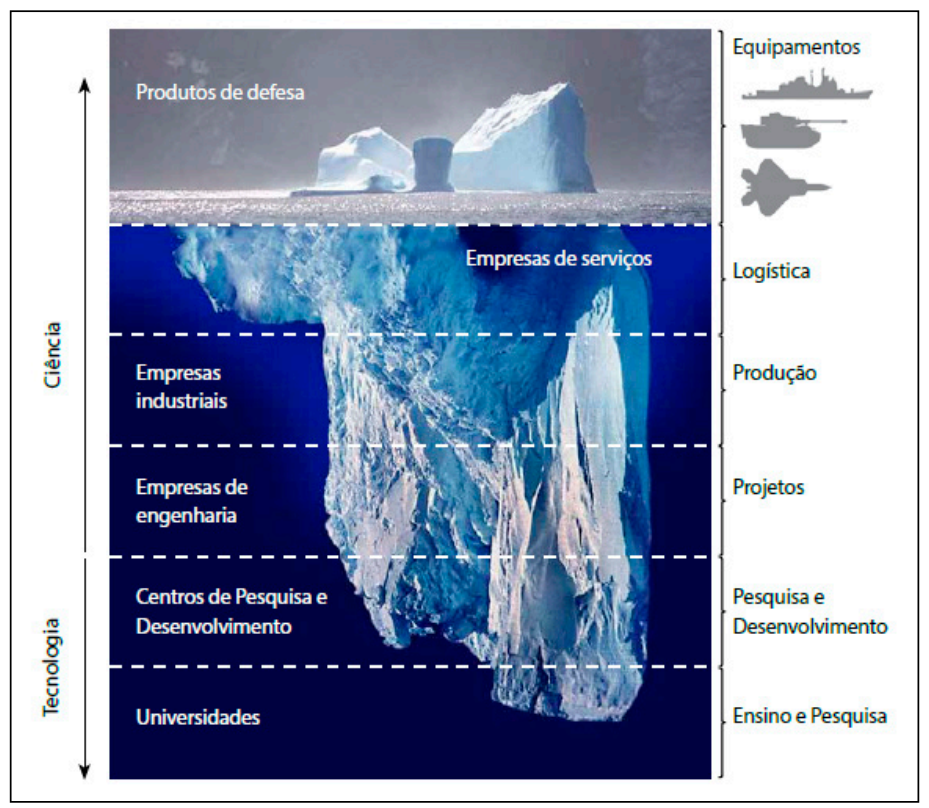

Figura 2 - Iceberg Científico Tecnológico de Defesa.

Fonte: LBDN (2012).

Esse iceberg científico vai ao encontro do trabalho sobre intensidade tecnológica de Furtado (1994), que afirma que o desenvolvimento de conhecimento tecnológico, pode ser adquirido através de institutos de pesquisa, laboratórios de universidades, fornecedores, dentre outros. Ou seja, as fontes para o desenvolvimento tecnológico podem ser diversas e com diferentes perfis; todavia, esse desenvolvimento pode surgir dentro da empresa, por seus colaboradores e capital intelectual, alinhado a investimentos internos. A Figura 2 demonstra a complexidade que é a composição de várias empresas e instituições, bem como seus relacionamentos conflitantes, os quais devem ser harmoniosos, produzindo bens e serviços necessários às forças armadas (Amarante 2012). Diante do elevado grau de intensidade tecnológica da BID, é importante entender até que ponto possíveis diferenças de desenvolvimento encontradas entre as competências das empresas da BID e das OI devem-se ao fato das empresas da BID estarem sujeitas a maiores desafios ou ao seu elevado grau tecnológico. Dessa forma, propõe-se a segunda hipótese desse trabalho: 


\section{H2: AS COMPETÊNCIAS DAS EMPRESAS DA BASE INDÚSTRIAL DE DEFESA (BID) SÃO MAIS DESENVOLVIDAS \\ DO QUE AS DAS EMPRESAS DE OUTRAS INDÚSTRIAS (OI), QUANDO OBSERVADAS APENAS AS COM BAIXA INTENSIDADE TECNOLÓGICA (OIBIT), MAS SEMELHANTES ÀS DE ALTA INTENSIDADE TECNOLÓGICA (OIAIT)}

\section{Competência Organizacional}

O teste das hipóteses propostas passa primeiramente por uma compreensão sobre o conceito de competência organizacional. Uma competência organizacional pode ser compreendida como habilidades e características intangíveis que uma organização concebe ao longo do tempo e que se torna perceptível aos olhos dos clientes (Hayes, Pisano, Upton, and Wheelwright 2008; Prahalad and Hamel 1990). A competência organizacional pode ser dispersa, estando atrelada a um conjunto de habilidades de uma unidade empresarial ou específica, referindo-se a uma função organizacional como produção, marketing ou gestão de projetos (Perunović, Christoffersen, and Mefford 2012).

Com base nesse conceito, pode-se dizer que empresas que atuam no ramo de entregas utilizam-se dos mesmos recursos, como veículos, centro de distribuição, pessoas e rotas. Contudo, alguns clientes optam por determinada empresa para realizar tal serviço porque essa conseguiu configurar seus recursos de forma a passar para esse cliente, que ela tem habilidades como rapidez, zelo no transporte e confiabilidade no prazo, melhores do que outras empresas, isto é, ela oferece uma competência diferenciada de entrega (Paiva, Carvalho Jr., and Fensterseifer 2009; Prahalad and Hamel 2005). Portanto, competências como essas advém da experiência da empresa em fabricar algo ou prestar um serviço, o que permite a ela crescimento e diversificação (Andrews 1980). Algumas competências com propriedades complexas podem ter mais valor e gerar maiores resultados para as empresas, especialmente aquelas que geram inovação ou têm potencial para tal (Almeida, Andrade, Alencar, Assis, and Silva 2016; Ferreira 2016). Em alguns casos, dependendo do contexto em que uma empresa está inserida, como em um projeto, tal oportunidade pode ser superior à sua competência, forçando-a a elevar o nível dessa competência ao seu grau máximo, desafiando sua estratégia (Andrews 1980). Por outro lado, é importante que as empresas realizem uma avaliação de suas competências, principalmente quando elas são estratégicas, pois é possível que determinados processos e métodos, possam ser adaptados e otimizados, de acordo com as necessidades de cada departamento interno (Markowitsch and Plaimauer 
2009). Apesar de difícil e complexa a gestão desses eventos, quando uma empresa consegue essa reconfiguração, priorizando competências específicas, ela acaba dando um salto sobre seus concorrentes e alcança vantagem competitiva (Andrews 1980; Hayes et al. 2008; Prahalad and Hamel 2005).

Cabe destacar que competências podem ser desenvolvidas e acumuladas, aperfeiçoando cada vez mais a qualidade das práticas e metodologias (Rigby 2013). Desenvolver e fomentar competências está ligado à aprendizagem em longo prazo da empresa, questão que afeta positivamente os processos internos desta, as relações entre as equipes de projeto, os planos futuros e o aumento dos níveis de desenvolvimento tecnológico (Danneels 2002). Dado o contexto específico que a BID demonstra ter e que em parte foi exposto nesse texto, é necessária uma análise sobre as competências desse segmento. Com base nos indícios propostos por Amarante (2012) e na metodologia utilizada por Bach, Conde-Molist, Ledoux, Matt e Schaeffer (1995) e Furtado e Costa Filho (2009) foram escolhidas as competências a serem analisadas nesse estudo. Para as competências relativas à melhoria contínua, flexibilidade/customização, gestão de fornecedores, desenvolvimento de produtos e serviços e pós-venda, todas as escalas são oriundas do trabalho de Scarpin (2016). Já as escalas relativas à gestão de indicadores, comunicação e integração, metas e objetivos, gestão de recursos humanos e gestão de projetos têm origem no trabalho de Bloom, Genakos, Sadun e Van Reenan (2012), com algumas adaptações baseadas em Scarpin (2016). A avaliação de mercado e responsividade mercadológica têm suas escalas baseadas em Kohli, Jaworski e Kumar (1993). A gestão de riscos teve suas variáveis retiradas do trabalho de Zwikael, Shimizu, e Globerson (2005). Por fim, a resiliência foi baseada em Wieland e Wallenburg (2012) e a aversão ao risco em Das e Joshi (2007).

\section{MÉTODO}

\section{População, amostras, escalas e coleta dos dados}

A coleta de dados desta pesquisa foi dividida em duas etapas. Primeiramente, foi abordado o conjunto de empresas que fazem parte da indústria brasileira em geral, denominada Outras Indústrias (OI), isto é, empresas que atuam em segmentos como têxtil, metalurgia, automotivo, maquinário, químico, construção civil e outros. Para a análise da segunda hipótese, este conjunto de empresas foi dividido em Outras Indústrias de Alta Intensidade Tecnológica (OIAIT) e Outras Indústrias de Baixa Intensidade Tecnológica (OIBIT). A pesquisa foi realizada em âmbito Brasil. O contato das empresas pesquisadas foi obtido através de uma ba- 
se de dados fornecida pelo Instituto de Pesquisas e Estudos Industriais (IPEI), que continha 4.033 empresas. Já na segunda etapa, foi abordado o conjunto de empresas que fazem parte da BID. Essas empresas foram contatadas através de uma lista obtida no site da Associação Brasileira das Indústrias de Materiais de Defesa e Segurança (ABIMDE), que continha 199 empresas. A amostra relativa às empresas de OI foi acessada através de um questionário eletrônico, reforçado por contatos telefônicos, que solicitavam a participação na pesquisa. Ao final desse processo, foram angariados 161 questionários respondidos, que, após filtro metodológico, resultaram em 118 respostas devidamente disponíveis para análises quantitativas. Situação semelhante se deu para a amostra de empresas da BID, que no final alcançou 37 questionários disponíveis para análise. A escala utilizada foi o modelo de Likert com 5 pontos, ancorados em "Discordo totalmente" e "Concordo totalmente".

\section{ANÁLISE DOS DADOS}

Para a análise dos dados deste trabalho, inicialmente são retomadas as hipóteses formuladas. A primeira hipótese propõe que as empresas da BID possuem competências mais desenvolvidas do que asempresas de OI. Já a segunda propõe que as empresas da BID têm competências mais desenvolvidas do que as empresas de baixa intensidade tecnológica de outras industrias (OIBIT) e, por outro lado, que as mesmas empresas da BID têm competências com desenvolvimento semelhante às empresas de alta intensidade tecnológica de outras indústrias (OIAIT). Desta forma, dado que as hipóteses formuladas objetivam verificar se existem diferenças entre os níveis das competências das empresas por indústria, foi empregada a Análise de Variância (ANOVA). Depois, foram realizadas comparações planejadas através de testes post hoc (Tukey e Games-Howell), buscando comparações que validem essas diferenças (Field 2009). No total, foram examinadas 15 competências, que juntas somavam 58 variáveis. Através da ANOVA foi possível identificar 14 variáveis de 10 competências com diferenças significativas em virtude da indústria a que pertencem. Destacam-se as empresas da BID com competências superiores às das OI, como Gestão de Projetos (50\%), ${ }^{1}$ Gestão de Riscos (50\%) e Avaliação de Mercado (40\%). 


\section{Tabela 1}

ANOVA sobre as Competências com diferenças por Indústria

\begin{tabular}{|c|c|c|c|c|c|}
\hline Competência & Variável & $\begin{array}{c}\text { ID } \\
(n=37)\end{array}$ & $\begin{array}{c}\text { OIAI } \\
(n=57)\end{array}$ & $\begin{array}{c}\text { OIBI } \\
(n=61)\end{array}$ & Sig. \\
\hline $\begin{array}{l}\text { Flexibilidade e } \\
\text { customização }\end{array}$ & $\begin{array}{l}\text { Customizamos nossos produtos/ } \\
\text { serviços para atender às demandas } \\
\text { dos clientes }\end{array}$ & 4,56 & 3,89 & 4,26 & 0,03 \\
\hline $\begin{array}{l}\text { Desenvolvimento } \\
\text { de produtos e } \\
\text { serviços }\end{array}$ & $\begin{array}{l}\text { Somos líderes na utilização de novas } \\
\text { tecnologias em nosso segmento }\end{array}$ & 4,16 & 3,32 & 3,46 & 0,01 \\
\hline $\begin{array}{l}\text { Gestão de } \\
\text { indicadores }\end{array}$ & $\begin{array}{l}\text { Dispomos de quadros atualizados } \\
\text { com indicadores de desempenho em } \\
\text { exposição pela empresa }\end{array}$ & 2,92 & 3,46 & 3,75 & 0,04 \\
\hline $\begin{array}{l}\text { Gestão de } \\
\text { recursos } \\
\text { humanos }\end{array}$ & $\begin{array}{l}\text { Funcionários com desempenho fraco } \\
\text { são dispensados ou alocados em } \\
\text { áreas menos críticas }\end{array}$ & 3,94 & 3,00 & 3,20 & 0,01 \\
\hline \multirow[t]{2}{*}{$\begin{array}{l}\text { Avaliação de } \\
\text { mercado }\end{array}$} & $\begin{array}{l}\text { Interagimos diretamente com os } \\
\text { clientes para entender a melhor } \\
\text { forma de servi-los }\end{array}$ & 4,73 & 4,26 & 4,05 & 0,00 \\
\hline & $\begin{array}{l}\text { Avaliamos periodicamente os efeitos } \\
\text { que mudanças no ambiente } \\
\text { competitivo podem ter em nossos } \\
\text { clientes }\end{array}$ & 3,73 & 3,12 & 3,73 & 0,02 \\
\hline $\begin{array}{l}\text { Responsividade } \\
\text { mercadológica }\end{array}$ & $\begin{array}{l}\text { Respondemos rapidamente a } \\
\text { mudanças da estrutura de preço da } \\
\text { concorrência }\end{array}$ & 3,64 & 3,07 & 3,65 & 0,03 \\
\hline \multirow[t]{3}{*}{$\begin{array}{l}\text { Gestão de } \\
\text { projetos }\end{array}$} & $\begin{array}{l}\text { Adotamos métodos tradicionais } \\
\text { de gestão de projetos (ex. WBS, } \\
\text { Gestão de requisitos) }\end{array}$ & 3,79 & 2,65 & 2,60 & 0,00 \\
\hline & $\begin{array}{l}\text { Existem métricas para mensurar o } \\
\text { desempenho dos projetos }\end{array}$ & 3,91 & 3,04 & 3,23 & 0,03 \\
\hline & $\begin{array}{l}\text { Utilizamos softwares de } \\
\text { gerenciamento de projetos (ex. } \\
\text { Ms-Project) }\end{array}$ & 3,81 & 3,11 & 2,63 & 0,00 \\
\hline \multirow[t]{2}{*}{ Gestão de riscos } & $\begin{array}{l}\text { Avaliamos sistematicamente } \\
\text { possíveis fontes de risco para a } \\
\text { nossa empresa }\end{array}$ & 3,89 & 3,18 & 3,62 & 0,05 \\
\hline & $\begin{array}{l}\text { Consideramos importante avaliar } \\
\text { os riscos da empresa e dos } \\
\text { fornecedores e clientes }\end{array}$ & 4,50 & 3,91 & 4,02 & 0,05 \\
\hline Resiliência & $\begin{array}{l}\text { Conseguimos ter um bom } \\
\text { desempenho independentemente da } \\
\text { situação do mercado }\end{array}$ & 2,86 & 3,54 & 3,60 & 0,01 \\
\hline Aversão ao risco & $\begin{array}{l}\text { Adotar estratégias que } \\
\text { gerem elevados custos }\end{array}$ & 2,03 & 2,75 & 2,78 & 0,00 \\
\hline
\end{tabular}

Fonte: Elaborado pelos autores. 
Os testes post hoc (Tabela 2 ) auxiliam na compreensão detalhada de cada diferença que a ANOVA sugeriu. Na variável que trata sobre a customização de produtos e serviços, percebe-se que as empresas da BID $(4,56)$ são superiores às empresas de $\operatorname{OIAIT}(3,89)$, mas semelhantes às empresas de OIBIT $(4,26)$. Em relação à utilização de novas tecnologias, a BID $(4,16)$ se sobrepõe em relação às duas indústrias, $\operatorname{OIAIT}(3,32)$ e $\operatorname{OIBIT}(3,46)$. Por outro lado, os quadros atualizados com indicadores de desempenho da empresa das empresas de OIBIT tem níveis melhores $(3,75)$ que a BID $(2,92)$ e semelhantes às OIAIT $(3,46)$. Sobre a realocação ou dispensa de funcionários com desempenho fraco, a BID $(3,94)$ tem diferença tanto para as $\operatorname{OIAIT}(3,00)$ como para as $\operatorname{OIBIT}(3,20)$. Em relação à interação com o cliente, a BID $(4,73)$ também se difere das OIAIT $(4,26)$ e OIBIT $(4,05)$. Já a avaliação sobre efeitos no ambiente competitivo, tanto a $\operatorname{BID}(3,73)$ como as $\operatorname{OIBIT}(3,73)$ são melhores que as OIAIT $(3,12)$, ambas compõem a Avaliação de Mercado. Sobre as respostas frente à estrutura de preço da concorrência, tanto a BID $(3,64)$ quanto as $\operatorname{OIBIT}(3,65)$ são melhores que as $\operatorname{OIAIT}(3,07)$.

Analisando a Gestão de Projetos, na primeira variável sobre a adoção de métodos tradicionais a BID $(3,79)$ supera as OIAIT $(2,65)$ e OIBIT $(2,60)$. Sobre a existência de métricas para mensurar o desempenho dos projetos, a BID $(3,91)$ novamente se sobrepõe às $\operatorname{OIAIT}(3,04)$ e às $\operatorname{OIBIT}(3,23)$. Já sobre o uso de softwares para auxílio no gerenciamento, a $\operatorname{BID}(3,81)$ se diferencia apenas em relação às $\operatorname{OIBIT}(2,63)$, sem diferença para as OIAIT $(3,11)$. Sobre a Gestão de Riscos, no caso, a primeira variável sobre a avaliação de fontes de risco, a BID tem resultados melhores $(3,89)$ em relação às OIAIT $(3,18)$, mas semelhantes em relação às OIBIT $(3,62)$. Na segunda variável sobre a importância de se avaliar riscos, a BID $(4,50)$ é melhor apenas quando comparada às OIAIT $(3,91)$, sem diferenças para as OIBIT $(4,02)$. Sobre o bom desempenho independentemente da situação do mercado, tanto as OIAIT $(3,54)$ como as $\operatorname{OIBIT}(3,60)$ são melhores que a $\operatorname{BID}(2,86)$. E, finalmente, no que diz respeito à adoção de estratégias que gerem elevados custos, novamente OIAIT $(2,75)$ e OIBIT $(2,78)$ têm melhores e diferentes médias do que a $\operatorname{BID}(2,03)$. 


\section{Tabela 2}

Testes post hoc sobre as competências com diferenças por Indústria

\begin{tabular}{|c|c|c|}
\hline \multicolumn{3}{|c|}{ Base Industrial de Defesa (BID) > Outras Indústrias (OI) } \\
\hline Competência & Variável & Hipótese 1 \\
\hline $\begin{array}{l}\text { Desenvolvimento de produtos } \\
\text { e serviços* }\end{array}$ & $\begin{array}{l}\text { Somos líderes na utilização de novas } \\
\text { tecnologias em nosso segmento }\end{array}$ & \multirow{5}{*}{ Confirmada } \\
\hline Gestão de recursos humanos & $\begin{array}{l}\text { Funcionários com desempenho fraco } \\
\text { são dispensados ou alocados em } \\
\text { áreas menos críticas }\end{array}$ & \\
\hline Avaliação de mercado* & $\begin{array}{l}\text { Interagimos diretamente com os } \\
\text { clientes para entender a melhor forma } \\
\text { de servi-los }\end{array}$ & \\
\hline Gestão de projetos* & $\begin{array}{l}\text { Adotamos métodos tradicionais de } \\
\text { gestão de projetos (ex. WBS, Gestão de } \\
\text { requisitos) }\end{array}$ & \\
\hline Gestão de projetos* & $\begin{array}{l}\text { Existem métricas para mensurar o } \\
\text { desempenho dos projetos }\end{array}$ & \\
\hline \multicolumn{3}{|c|}{$\begin{array}{c}\text { Base Industrial de Defesa (BID) > Outras Indústrias de Baixa Intensidade } \\
\text { Tecnológica (OIBI) }\end{array}$} \\
\hline Competência & Variável & Hipótese 2 \\
\hline Gestão de projetos* & $\begin{array}{l}\text { Utilizamos softwares de gerenciamento } \\
\text { de projetos (ex. Ms-Project) }\end{array}$ & Confirmada \\
\hline \multicolumn{3}{|c|}{$\begin{array}{c}\text { Base Industrial de Defesa (BID) } \leq \text { Outras Indústrias de Baixa Intensidade } \\
\text { Tecnológica }(\mathrm{OIBI})\end{array}$} \\
\hline Competência & Variável & Hipótese 2 \\
\hline Gestão de indicadores & $\begin{array}{l}\text { Dispomos de quadros atualizados } \\
\text { com indicadores de desempenho em } \\
\text { exposição pela empresa }\end{array}$ & \multirow{4}{*}{$\begin{array}{l}\text { Efeito } \\
\text { contrário do } \\
\text { previsto }\end{array}$} \\
\hline Flexibilidade e customização* & $\begin{array}{l}\text { Customizamos nossos produtos/ } \\
\text { serviços para atender às demandas } \\
\text { dos clientes }\end{array}$ & \\
\hline \multirow[b]{2}{*}{ Gestão de riscos } & $\begin{array}{l}\text { Avaliamos sistematicamente possíveis } \\
\text { fontes de risco para a nossa empresa }\end{array}$ & \\
\hline & $\begin{array}{l}\text { Consideramos importante avaliar os } \\
\text { riscos da empresa e dos fornecedores e } \\
\text { clientes }\end{array}$ & \\
\hline
\end{tabular}




\begin{tabular}{|c|c|c|}
\hline \multicolumn{3}{|c|}{$\begin{array}{c}\text { Base Industrial de Defesa (BID) } \geq \text { Outras Indústrias de Alta Intensidade } \\
\text { Tecnológica (OIAI) }\end{array}$} \\
\hline Competência & Variável & Hipótese 2 \\
\hline Gestão de projetos* & $\begin{array}{l}\text { Utilizamos softwares de gerenciamento } \\
\text { de projetos (ex. Ms-Project) }\end{array}$ & Confirmada \\
\hline \multirow[b]{2}{*}{ Gestão de riscos } & $\begin{array}{l}\text { Avaliamos sistematicamente possíveis } \\
\text { fontes de risco para a nossa empresa }\end{array}$ & \multirow{3}{*}{$\begin{array}{l}\text { Efeito } \\
\text { contrário do } \\
\text { previsto }\end{array}$} \\
\hline & $\begin{array}{l}\text { Consideramos importante avaliar os } \\
\text { riscos da empresa e dos fornecedores e } \\
\text { clientes }\end{array}$ & \\
\hline Flexibilidade e customização* & $\begin{array}{l}\text { Customizamos nossos produtos/ } \\
\text { serviços para atender às demandas } \\
\text { dos clientes }\end{array}$ & \\
\hline \multicolumn{3}{|c|}{$\begin{array}{l}\text { Base Industrial de Defesa (BID) }=\text { Outras Indústrias de Baixa Intensidade } \\
\text { Tecnológica }(\text { OIBI })>\text { Outras Indústrias de Alta Intensidade Tecnológica (OIAI) }\end{array}$} \\
\hline Competência & Variável & Hipótese 2 \\
\hline Avaliação de mercado** & $\begin{array}{l}\text { Periodicamente avaliamos os efeitos } \\
\text { que mudanças no ambiente competitivo } \\
\text { podem ter em nossos clientes }\end{array}$ & \multirow{2}{*}{$\begin{array}{l}\text { Efeito } \\
\text { contrário do } \\
\text { previsto }\end{array}$} \\
\hline $\begin{array}{l}\text { Responsividade } \\
\text { mercadológica }\end{array}$ & $\begin{array}{l}\text { Respondemos rapidamente a mudanças } \\
\text { da estrutura de preço da concorrência }\end{array}$ & \\
\hline
\end{tabular}

Nota: *Aplicação do teste de Games-Howell $\operatorname{com} p=0,05$, dada a igualdade de variâncias não assumidas.

Fonte: Elaborado pelos autores.

Os resultados da Tabela 2 sugerem que algumas competências têm maior desenvolvimento na BID, ambas relacionadas ao Desenvolvimento de Produtos e Serviços, Gestão de Recursos Humanos, Avaliação de Mercado e à Gestão de Projetos. No entanto, esses resultados não são determinísticos, dado que poucas variáveis expuseram tais diferenças, podendo-se excetuar, em parte, apenas a gestão de projetos, pois a mesma figura com diferenças significativas em três de seis variáveis que a compõe. Portanto, a primeira hipótese deste estudo é confirmada em parte, dado que poucas competências da BID tiveram resultados acima de OI, sendo que, dessas, apenas a gestão de projetos teve resultados relevantes. Por outro lado, os resultados desse estudo não tiveram subsídios mínimos para sustentar sequer uma discussão sobre a segunda hipótese, a qual presumia que resultados iguais ou sem diferença significativa entre BID e OIAIT aparecessem para diversas competências e variáveis, e não somente para uma, como ocorrido nesse trabalho - uso de softwares de gerenciamento de projetos (ex. Ms-Project). 


\section{DISCUSSÃO E CONSIDERAÇÕES FINAIS}

O presente artigo buscou, por meio de uma análise quantitativa, evidenciar possíveis diferenças entre os níveis de desenvolvimento das mesmas competências de grupos de empresas distintas, isto é, Base Industrial de Defesa (BID) x Outras Indústrias de Alta Intensidade Tecnológica (OIAIT) X Outras Indústrias de Baixa Intensidade Tecnológica (OIBIT). Desta forma, buscou-se evidenciar se as indústrias de diferentes áreas de atuação influenciam em tais níveis ou até mesmo em competências próprias ou necessárias em determinada indústria. Analisando-se apenas o valor das médias das competências, organizadas pela indústria especifica a que pertencem, percebem-se valores distintos. No entanto, foi através da aplicação da ANOVA e de testes post hoc (Sig. $\leq$ 0,05) que foi possível descobrir se essas diferenças eram significativas ou não. Nessas análises, apareceram 14 variáveis de 10 competências com diferenças significativas.

Das 14 variáveis, apenas em 5 delas a BID demonstra ter resultados melhores que as outras indústrias (OIAI e OIBI). São variáveis relacionadas ao uso de novas tecnologias, realocação ou dispensa de funcionários com desempenho fraco, interação direta com os clientes, adoção de métodos tradicionais de gestão de projetos e existência de métricas para mensuração do desempenho de projetos. Esses resultados, quando comparados à literatura (cf. Amarante 2012; Freitas 2011), parecem fazer sentido, dado o caráter específico e inerente às características que compõem projetos como os que são realizados na BID. A variável sobre uso de softwares no gerenciamento de projetos em parte corrobora essa premissa, dado que a BID teve resultados melhores apenas em relação às OIBIT e parecidos com as OIAIT.

Por outro lado, em relação ao nível de desenvolvimento devariáveis como customização de produtos, avaliação de efeitos no ambiente competitivo, respostas diante de estruturas de preço da concorrência, avaliação de fontes de riscos e importância de se avaliar riscos, os resultados surpreendem dado que a BID mostrou ser superior as OIAIT e semelhantes às empresas de OIBIT. Esses resultados sugerem que as OIAIT têm tido dificuldades na manutenção de competências que são importantes em atividades de alta intensidade tecnológica, ou que as empresas de OIBIT tem aprimorado competências que podem lhes trazer vantagem competitiva no futuro.

Por último, em relação à existência de quadros atualizados com indicadores de desempenho pela empresa, as OIBIT e OIAIT têm resultados melhores que a BID, sugerindo que detalhes de organização básica e acompanhamento da rotina organizacional precisam ser aprimorados na BID, sendo que nas OI continua sendo uma prática padrão bem assentada. Já o bom desempenho independentemente da situação de mercado e a adoção de estratégias 
que gerem elevados custos são competências em que as OI conseguem ter resultados melhores que a BID, naturalmente pela dinâmica mercadológica e econômica diferenciada que as empresas da BID vivenciam (Amarante 2012; ABDI 2011). Neste caso, para que esses resultados mudem em favor da BID, sugere-se a implementação de políticas públicas e novas práticas que tragam estabilidade nas relações entre governo e empresas da BID.

Apesar das competências Gestão de Riscos (50\%) e Avaliação de Mercado $(40 \%)$ terem surgido com boa parte de suas variáveis com resultados melhores em prol da BID, há de se destacar a competência Gestão de Projetos, pois esta aparece com as 3 principais de 6 variáveis $(50 \%)$, com diferenças significativas também em favor das empresas da BID. As outras três variáveis dizem respeito à I) adoção de práticas de gestão de riscos em todos os projetos, II) busca por novas práticas e ferramentas de gestão de projetos e III) programas para qualificar funcionários em gestão de projetos. Essas não tiveram diferenças entre as indústrias analisadas, sugerindo-se que essas questões são trabalhadas com desempenhos próximos, independentemente da indústria.

A evidência de um melhor desempenho da Gestão de Projetos em favor das empresas da BID sentido é coerente, pois grande parte dos produtos e serviços dessa indústria são projetos (p. ex. helicópteros, aeronaves, submarinos, sistemas de defesa, etc.), dos quais a maioria possui alto grau de complexidade, demandando desenvolvimentos em ciclos longos, com baixa frequência de aquisição e custos de mudança e transação altos, que na maioria das vezes são encomendados pelos governos, sendo que um fornecimento desse nível necessita de equipes de trabalho engajadas e técnicas, especialmente em gestão de projetos (Amarante 2012; Markusen 1986).

Mudanças tecnológicas e o novo padrão de eficiência empregado na competição entre empresas fizeram com que, nos últimos anos, a gestão de projetos despertasse o interesse de pesquisadores em gestão, especialmente pelo fato dela estar ligada a diversos aspectos organizacionais como habilidades para a resolução de problemas complexos, desenvolvimento pessoal e relacionamento entre equipes multidisciplinares, colocando-se, dessa forma, como um novo critério qualificador de uma empresa (Maylor 2001). Um fato interessante é que a gestão de projetos nasceu e se desenvolveu justamente na Indústria de Defesa e na Construção Civil (Hayes et al. 2008; Maylor 2001). Outra característica recorrente em projetos é a questão da complexidade, presente também na indústria cinematográfica (Slack, Brandon-Jones, and Johnston 2018). De acordo com Pollock $(2015,13)$, relembrando comentários do diretor de cinema George Lucas, "Fazer um filme é como apagar um incêndio com uma peneira. Há tantos elementos, e ele vai ficando cada vez mais complicado”.

Logo, entende-se que a gestão de projetos é uma atividade exigente e multifuncional, que busca atingir objetivos de um determinado proje- 
to. Requerendo habilidades como conhecimento técnico e habilidades interpessoais, que devem ser empregadas em atividades com quantidade de recursos definida e plano de trabalho com começo, meio e fim, devendo, ainda, tirar proveito da estrutura organizacional e de todas as ferramentas e técnicas de gestão disponíveis, sem prejuízo da rotina de operações da empresa (Munns and Bjeirmi 1996). Cabe destacar que um projeto é permeado por decisões complexas e antagônicas que se balizam em questões como custo, tempo e qualidade, onde o êxito dessa competência está na adequada administração dos recursos, que, na maioria das vezes, são críticos (Atkinson 1999; Jacobs and Chase 2009).

Interessante que essa competência parece estar ligada à qualidade dos recursos humanos das empresas, pois somente a existência de pessoal capacitado viabiliza um trabalho exitoso no desenvolvimento de meios, sistemas, equipamentos e serviços militares (Cunha and Amarante 2011). De acordo com Markusen (1986) e Schimdt e Assis (2013) a importância do capital humano é explícita na BID, pois o setor é dependente de pessoas com alto grau de qualificação, com conhecimentos sobre gestão, projetos, inovação, química, engenharia e outros. Logo, políticas educacionais e de formação de pessoas podem ser pensadas para o desenvolvimento e expansão da BID no Brasil.

A gestão de projetos concede às empresas a oportunidade de construir uma competência, que pode tornar-se essencial, principalmente quando atuam na integração de sistemas (Davies, Brady, and Hobday 2007). Cabe frisar a dificuldade para a construção dessa competência, que tem suas raízes na capacidade da empresa absorver informações e conhecimentos existentes nos ambientes interno e externo ao projeto (Lampel 2001). Em qualquer indústria é difícil desenvolver a competência em gestão de projetos. Percebe-se nos dias atuais que menos de duas dezenas de países têm essa competência para projetar, construir e equipar, de forma autônoma, diversos e tecnológicos produtos de defesa (Amarante 2012).

Diante dos achados nota-se que a Gestão de Projetos na BID tem de lidar com um cenário de diversidades e incerteza diferente das OI, trabalhando com aprendizagem, ampliação e reconfiguração de recursos materiais/humanos e competências funcionais, sendo que esse esforço na maioria das vezes deve resultar em produtos e serviços com relevante teor de inovação, que podem ser adaptados para o mercado civil — características essas semelhantes ao conceito de competência essencial criado no início de 1990. Segundo Prahalad e Hamel (1990; 2005), a competência essencial diz respeito a I) Aprendizado coletivo, coordenação de habilidades de produção e integração de tecnologias; II) Organização do trabalho e entrega de valor; III) Comunicação, envolvimento e comprometimento de equipes e pessoas de diferentes departamentos; IV) Desenvolve-se com o passar 
do tempo, desde que aplicada e compartilhada; V) Dessa forma, é difícil de ser copiada/ imitada, pelo menos a curto prazo; e VI) Promove o acesso da empresa a outros mercados e a difusão de inovação. Assim, o presente estudo argumenta que a competência de Gestão de Projetos na BID reúne essas qualidades (cf. Hayes et al. 2008; Lampel 2001; Maylor 2001; Munns and Bjeirmi 1996), implicando em níveis maiores de desenvolvimento dada a sua importância, melhoria contínua e singularidade na BID, permitindo que esta seja uma competência essencial nessa indústria.

\section{LIMITAÇÕES E SUGESTÕES PARA FUTURAS PESQUISAS}

Apesar dos poucos estudos na esfera militar e na BID que tratam sobre competências organizacionaise considerando os achados dessa pesquisa, esse trabalho reconhece algumas limitações. A primeira delas relacionada ao caráter exploratório, no qual pretendia-se descobrir possíveis diferenças em relação deão nível de desenvolvimento das competências das empresas abordadas em indústrias distintas. Consequentemente, a técnica de análise estatística utilizada acompanha o objetivo de exploração, demonstrando tais diferenças, mas não conseguindo explicar o porquê delas. Os níveis superiores de desenvolvimento da gestão de projetos expostos nas análises dão indícios de que esta seja uma competência essencial na BID; todavia, não as confirmam, necessitando de novos estudos.

Em relação às futuras pesquisas, outros trabalhos poderiam aprofundar o exame da gestão de projetos como competência essencial na BID, utilizando-se de novas comparações, p. ex., abordando-a como uma variável independente e sua influencia sobre os outputs possíveis (Prahalad e Hamel 1990). Outra oportunidade de pesquisa seria a avaliação das competências que tiveram melhores resultados no que diz respeito às empresas de OI, isto é, o porquê de elas serem mais desenvolvidas, e como a BID deve avaliar essas competências, decidindo por aperfeiçoar ou não as suas.

\section{AGRADECIMENTOS}

Os autores agradecem o Conselho Nacional de Desenvolvimento Tecnológico e Científico (CNPq) e o Instituto Pandiá Calógeras pela concessão da bolsa de pesquisa sob n. ${ }^{\circ}$ 471637-2014-9. Os órgãos de financiamento não estavam envolvidos na execução da pesquisa. Somos também gratos pela colaboração dos órgãos públicos, empresas, especialistas do setor e, sobretudo pelos comentários e sugestões dos revisores da RBED e dos colegas Alexandre L. Prim e Bruna Renata S. Leão. 


\section{REFERÊNCIAS}

Agência Brasileira de Desenvolvimento Industrial. 2011. Diagnóstico: Base Industrial de Defesa Brasileira, no. 54, 1. Campinas: ABDI, NEIT-IE-UNICAMP.

Almeida, L. C. P., E. P. Andrade, R. S. Alencar, W. S. de Assis, and A. M. da Silva. 2016. "Inovação em instituição militar de pesquisa: um estudo de caso exploratório”. Revista Produção Online 16, no. 4, 1371.

Amarante, J. C. A. 2012. A base industrial de defesa brasileira. Texto para Discussão. Rio de Janeiro: Instituto de Pesquisa Econômica Aplicada (IPEA).

Andrews, K. R. 1980. The concept of corporate strategy. New York: Dow JonesIrwin.

Atkinson, R. 1999. Project management: cost, time and quality, two best guesses and a phenomenon, it's time to accept other success criteria. International journal of project management 17 , no. 6, 337-342.

Bach, L., N. Conde-Molist, M. J. Ledoux, M. Matt, and V. Schaeffer. 1995. "Evaluation of the economic effects of Brite-Euramprogrammeson the European industry”. Scientometrics 34, no. 3, 325-349.

Bloom, N., C. Genakos, R. Sadun, and J. Van Reenen. 2012. "Management practices across firms and countries". The Academy of Management Perspectives 26, no. $1,12-33$.

Caldwell, N., and M. Howard. 2014. "Contracting for complex performance in markets of few buyers and sellers: The case of military procurement". International Journal of Operations \& Production Management 34, no. 2, 270-294.

Chiarini, T., and A. L. G. Silva. 2017. "Comércio exterior brasileiro de acordo com a intensidade tecnológica dos setores industriais: notas sobre as décadas de 1990 e 2000". Nova Economia 26, no. 3.

Corrêa, C. R., and C. H. Cagnin. 2016. "Prospective games for defense strategic decisions in Brazil”. Foresight 18, no. 1, 4-23.

Cunha, M. B., and J. C. A. Amarante. 2011. "O Livro Branco e a Base Científica, Tecnológica, Industrial e Logística de Defesa”. Revista da Escola de Guerra Naval 17, no. 1, 11. Rio de Janeiro: Escola de Guerra Naval.

Danneels, E. 2002. "The dynamics of product innovation and firm competences". Strategic Management Journal, Chicago 23, no. 12, 1095. 
Das, S. R., and M. P. Joshi. 2007. Process innovativeness in technology services organizations: Roles of differentiation strategy, operational autonomy and risk-taking propensity. Journal of Operations Management 25, no.3, 643-660.

Davies, A., T. Brady, and M. Hobday. 2007. "Organizing for solutions: Systems seller vs. systems integrator”. Industrial marketing management 36, no. 2, 183-193.

Duarte, É. E. 2012. Tecnologia militar e desenvolvimento econômico: Uma análise histórica. Texto para Discussão. Rio de Janeiro: Instituto de Pesquisa Econômica Aplicada (IPEA).

Dunne, J. P. 1995. “The defense industrial base”. In: Hartley, K., and T. Sandler. Handbook of Defence Economics 1. Elsevier: Netherland.

Eliasson, G. 2011. "Advanced purchasing, spillovers and innovative discovery". Journal of evolutionary economics 21, no. 1, 121-139.

Ferreira, M. J. B. 2016. "Plataforma aeronáutica militar". In: Mapeamento da Base Industrial de Defesa. ABDI - Agência Brasileira de Desenvolvimento Indutrial: Ipea - Instituto de Pesquisa Econômica Aplicada. Brasília.

Field, A. 2009. Descobrindo a estatística usando SPSS. Porto Alegre: Artmed.

Franko, P. 2014. "The Defense Acquisition Trilemma: The Case of Brazil". Strategic Forum 1, no. 284, 1-15. Washington.

Freitas, N. O. 2011 . "Instrumentos de desenvolvimento nacional". Revista da Escola de Guerra Naval 17, no. 1, 11. Rio de Janeiro: Escola de Guerra Naval.

Furtado, A. C. 1994. Capacitação tecnológica, competitividade e política industrial: uma abordagem setorial e por empresas líderes. Texto para Discussão. Rio de Janeiro: Instituto de Pesquisa Econômica Aplicada (IPEA).

Furtado, A. T., and R. de Q. Carvalho. 2005. "Padrões de intensidade tecnológica da indústria brasileira: um estudo comparativo com os países centrais". São Paulo em Perspectiva 19, no. 1, 70-84.

Furtado, A. T., and E. J. Costa Filho. 2009. "Avaliação dos impactos econômicos dos Programas ERJ 145 e EMBRAER 170/190". In: Montoro, Guilherme Castanho Franco, and Marcio Nobre Migon. Cadeia Produtiva Aeronáutica Brasileira: oportunidades e desafios. Rio de Janeiro: BNDES.

Graham, G., and G. Hardaker. 1998. "Defense sector procurement and supply chain relationships". Supply Chain Management: An International Journal 3, no. 3, 142-148. 
Hayes, R., G. Pisano, D. Upton, and S. Wheelwright. 2008. Produção, estratégia e tecnologia: em busca da vantagem competitiva. Porto Alegre: Bookman.

Jacobs, F. R., and R. B. Chase. 2009. Administração da produção e operações: o essencial. Porto Alegre: Bookman.

Kohli, A. K., B. J. Jaworski, and A. Kumar. 1993. "MARKOR: a measure of market orientation”. Journal of Marketing research 30, no. 4, 467-477.

Lampel, J. 2001. "The core competencies of effective project execution: the challenge of diversity". International Journal of Project Management 19, no. 8, 471-483.

Livro Branco de Defesa Nacional. 2012. http://www.defesa.gov.br/arqui$\operatorname{vos} / 2012 / \mathrm{mes} 07 / \mathrm{lbdn} . p d f$

Markowitsch, J., and C. Plaimauer. 2009. "Descriptors for competence: towards an international standard classification for skills and competences". Journal of European Industrial Training 33, no. 8/9, 817-837.

Markusen, A. R. 1986. "Defence spending: a successful industrial policy?". International Journal of Urban and Regional Research 10, no. 1, 105-122.

Maylor, H. 2001. "Beyond the Gantt chart: Project management moving on". European Management Journal 19, no. 1, 92-100.

Mendes, M. G. 2010. "Política de defesa do Brasil." Nação e Defesa. Primavera, no. $125-4 .^{a}$ Série, $55-70$.

Munns, A. K., and B. F. Bjeirmi. 1996. "The role of project management in achieving project success". International journal of project management 14, no. 2, 81-87.

Paiva, E. L., J. M. Carvalho Jr., and J. E. Fensterseifer. 2009. Estratégia de produção e de operações: conceitos, melhores práticas, visão de futuro. Porto Alegre: Bookman.

Peng, G. 2004. Defensa nacional de China. China: China Intercontinental Press.

Perunović, Z., M. Christoffersen, and R. N. Mefford. 2012. "Deployment of vendor capabilities and competences throughout the outsourcing process". International Journal of Operations \& Production Management 32, no. 3, 351-374.

Pollock, D. 2015. George Lucas: Skywalking - a vida e a obra do criador de Star Wars. São Paulo: Évora.

Prahalad, C. K., and G. Hamel. 1990. "The core competence of the corporation". Harvard Business Review 68, no. 3, 79-91. 
2005. Competindo pelo futuro: estratégias inovadoras para obter o controle do seu setor e criar os mercados de amanhã. Rio de Janeiro: Elsevier.

Rauen, A. T. 2015. "Compras públicas de P\&D no Brasil: o uso do artigo 20 da Lei de Inovação”. Radar 40, no. 1, 7-17.

Rigby, J. 2013. Review of Pre-commercial Procurement Approaches and Effects on Innovation. Working Paper (Nov.), Manchester Institute of Innovation Research, University of Manchester, England. https://www.nesta.org.uk/sites/default/files/review_of_pre-commercial_procurement_approaches_and_effects_on_innovation_revised_ajr-14-11-2013_final.pdf

Scarpin, M. R. S. 2016. Operational capabilities' typology: an evolution from operational practices. Doctoral Dissertation, Escola de Administração de Empresas Fundação Getulio Vargas, São Paulo, Brasil.

Schmidt, F. H. de., and L. R. S. Assis. 2013. A dinâmica recente do setor de defesa no Brasil: Análise das características e do envolvimento das firmas contratadas. Texto para Discussão. Rio de Janeiro: Instituto de Pesquisa Econômica Aplicada (IPEA).

Slack, N., A. Brandon-Jones, and R. Johnston. 2018. Administração da produção. São Paulo: Atlas.

The Economist. 2016. Rocketing around the world: Weapons-makers reckon missiles will be their next big hit. https://www.economist.com/news/business/21702207-weapons-makers-reckon-missiles-will-be-their-next-big-hit-rocketing-around-world

Wieland, A., and C. Marcus Wallenburg. 2012. Dealing with supply chain risks: Linking risk management practices and strategies to performance. International Journal of Physical Distribution \& Logistics Management 42, no. 10, 887-905.

Zwikael, O., K. Shimizu, and S. Globerson. 2005. Cultural differences in project management capabilities: A field study. International Journal of Project Management 23 , no. $6,454-462$. 


\section{NOTAS}

1. Estes percentuais referem-se ao número de variáveis que compõem a competência e que tiveram diferenças significativas. Isto é, no caso da gestão de projetos, que tem seis variáveis, três delas tiveram essas diferenças em razão da análise por indústria e assim sucessivamente.

2. George Lucas dirigiu o primeiro filme da trilogia Star Wars (1977). Essa experiência foi tão estressante que ele só voltaria a dirigir 22 anos depois, no quarto filme, Star Wars — A ameaça fantasma (1999). 


\section{A INDÚSTRIA DE DEFESA NO BRASIL: \\ INVESTIGANDO A COMPETÊNCIA ESSENCIAL}

\section{RESUMO}

O desenvolvimento e produção de produtos e serviços de defesa envolve elevada intensidade tecnológica, demandando diferentes competências organizacionais das empresas da Base Industrial de Defesa (BID). Este estudo compara o nível de desenvolvimento das competências das empresas da BID e de outras indústrias para identificar as competências organizacionais essenciais para competir neste segmento.

Palavras-chave: Base Industrial de Defesa (BID); Competências organizacionais; Intensidade tecnológica; Gestão de Projetos.

\section{ABSTRACT}

The development and production of defense products and services involve high technological intensity, demanding different skills from firms in the Defense industry. This study compares the level' development of the competencies of companies in the Defense and other industries to understand the organizational competencies necessary to compete in this segment.

Keywords: Defense Industrial Base (DIB); Organizational competencies; Project Management; Technological intensity.

Recebido em 31/01/2018. Aceito para publicação em 23/02/2019. 
